\title{
(6) OPEN ACCESS \\ Development and validation of nomogram based on SIRI for predicting the clinical outcome in patients with nasopharyngeal carcinomas
}

\author{
Yuan Chen, ${ }^{1}$ Wenjie Jiang, ${ }^{1}$ Dan $\mathrm{Xi}_{1}{ }^{1}$ Jun Chen, ${ }^{2}$ Guoping Xu, ${ }^{1}$ Wenming Yin, ${ }^{1}$ \\ Junjun Chen, ${ }^{1}$ Wendong $\mathrm{Gu}^{1}$
}

'Department of Radiation Oncology, The Third Affiliated Hospital of Soochow University, Changzhou,

China

${ }^{2}$ Department of Respiratory, The Seventh People's Hospital of Changzhou Changzhou, China

\section{Correspondence to}

DrWendong Gu, Department of Radiation Oncology, The Third Affiliated Hospital of Soochow University, Changzhou 213003, China: guwendong1415@czfph. com

YC and WJ contributed equally.

Accepted 22 July 2018 Published Online First 20 August 2018

\section{Check for updates}

(C) American Federation for Medical Research 2019. Re-use permitted under CC BY-NC. No commercial re-use. Published by BMJ.

To cite: Chen $Y$, Jiang $W$, Xi D, et al. J Investig Med 2019;67:691-698.

\section{ABSTRACT}

The Systemic Inflammation Response Index (SIRI), based on peripheral lymphocyte, neutrophil, and monocyte counts, was recently investigated as a prognostic marker for several tumors. However, use of the SIRI has not been reported for nasopharyngeal carcinoma (NPC). We evaluated the prognostic value of the SIRI in primary and validation cohorts. We also established an effective prognostic nomogram for NPC based on clinicopathological parameters and the SIRI. The predictive accuracy and discriminative ability of the nomogram were determined using the concordance index (C-index) and a calibration curve and were compared with tumor-node-metastasis classifications. Our KaplanMeier survival analysis results showed that the SIRI was associated with the overall survival of patients with NPC in the primary and validation cohorts. The SIRI was identified to be an independent prognostic factor for NPC. In addition, we developed and validated a new prognostic nomogram that integrated clinicopathological factors and the SIRI. This nomogram can efficiently predict the prognosis of patients with NPC. The SIRI is a novel, simple and inexpensive prognostic predictor for patients with NPC. The SIRI has important value for predicting the prognosis of patients with NPC and developing individualized treatment plans.

\section{INTRODUCTION}

Nasopharyngeal carcinoma (NPC) is a type of malignant tumor originating from the nasopharyngeal mucosal epithelium and has obvious regional and epidemic characteristics. ${ }^{1}$ NPC occurs frequently in South China, and the incidence rate of NPC is higher in males than in females; NPC occurrence typically peaks in people aged 50-60 years. ${ }^{1}$ The pathogenesis of NPC remains unclear, and it is currently thought to be associated with Epstein-Barr virus (EBV) infection, family genetic factors and environmental factors. ${ }^{2}$ Because of the hidden location of the nasopharynx and the atypical early clinical symptoms of NPC, as well as its special pathological classification and the involvement of the lymphatic pathway, NPC is prone to local lymph node metastasis and distant metastasis. Radiation therapy is the preferred

\section{Significance of this study}

What is already known about this subject?

- In recent years, many studies have integrated two types of white blood cell indicators; the neutrophil lymphocyte ratio (NLR) and monocyte lymphocyte ratio (MLR) are considered to be independent prognostic factors for nasopharyngeal carcinoma (NPC), and their prognostic value is greater than that of only white blood cells.

What are the new findings?

- Systemic Inflammation Response Index (SIRI) was associated with the overall survival (OS) of patients with NPC in the primary and validation cohorts. The SIRI was identified to be an independent prognostic factor for NPC.

How might these results change the focus of research or clinical practice?

- This nomogram based on SIRI can efficiently predict the prognosis of patients with NPC. The SIRI is a novel, simple and inexpensive prognostic predictor for patients with NPC. The SIRI has important value for predicting the prognosis of patients with NPC and developing individualized treatment plans.

treatment method for NPC, and patients with locally advanced NPC should be treated with concurrent chemotherapy or neoadjuvant therapy combined with concurrent chemotherapy. However, once patients with NPC suffer from distant metastasis, their prognosis will be poor. ${ }^{3}$ The prognosis evaluation of NPC is based on the tumor-node-metastasis (TNM) staging criteria developed by the American Joint Cancer Committee (AJCC). However, TNM staging determines the clinical staging based on the anatomical structures of tumor invasion and cannot adequately reflect the biological heterogeneity of tumors. Patients with the same TNM staging have considerable differences in prognoses. ${ }^{4}$ Therefore, finding effective and 
accurate prognostic indicators and developing individualized treatment programs will likely extend the survival time of patients with NPC and be of significance to them.

Tumor prognosis is related to not only the local characteristics of the tumor but also the body's immune/inflammatory response. ${ }^{5}$ The inflammatory response leads to chronic oxidative stress and produces oxygen free radicals, both of which are significantly related to oncogenesis and progression. ${ }^{7}$ First, inflammation is an important part of the tumor microenvironment. Studies have shown that leucocyte infiltration exists continuously in NPC lesions, and the infiltrated leucocytes promote the growth of NPC cells. ${ }^{8}$ In addition, EBV infection is closely related to the development of NPC, and EBV-encoded RNAs can induce inflammatory responses and play an important role in NPC progression. ${ }^{9}$ In addition, a large number of cytokines and inflammation-related parameters can be monitored in the peripheral blood of patients with NPC. ${ }^{8}$ Therefore, inflammation is closely related to the biological behavior of NPC, and using inflammation-related parameters to predict the prognosis of NPC is an important molecular-based strategy. Conventional systemic inflammatory response indicators are white cell counts and acute reactive proteins. It has been reported that white cell counts, which mainly include neutrophils, lymphocytes, and monocytes, have prognostic value in NPC. ${ }^{10-13}$ In recent years, many studies have integrated two

Table 1 Baseline characteristics for patients with SIRI $\leq 0.84$ versus SIRI $>0.84$ in primary and validation cohort

\begin{tabular}{|c|c|c|c|c|c|c|c|c|}
\hline \multirow[b]{2}{*}{ Clinical parameter } & \multicolumn{4}{|l|}{ Primary cohort } & \multicolumn{4}{|c|}{ Validation cohort } \\
\hline & SIRI $\leq 0.84(106)$ & SIRI>0.84 (179) & $\chi^{2}$ & $P$ values & SIRI $\leq 0.84(81)$ & SIRI >0.84 (132) & $\chi^{2}$ & $P$ values \\
\hline \multicolumn{9}{|l|}{ Sex } \\
\hline Male & 69 & 141 & 6.42 & 0.011 & 50 & 107 & 9.68 & 0.002 \\
\hline Female & 37 & 38 & & & 31 & 25 & & \\
\hline \multicolumn{9}{|l|}{ Age } \\
\hline$<50$ & 40 & 66 & 0.02 & 0.884 & 33 & 53 & 0.01 & 0.932 \\
\hline$\geq 50$ & 66 & 113 & & & 48 & 79 & & \\
\hline \multicolumn{9}{|l|}{ BMI } \\
\hline$\leq 25$ & 71 & 138 & 3.48 & 0.062 & 56 & 103 & 2.10 & 0.147 \\
\hline$>25$ & 35 & 41 & & & 25 & 29 & & \\
\hline \multicolumn{9}{|l|}{ T stage } \\
\hline $\mathrm{T} 1$ & 23 & 24 & 4.50 & 0.212 & 11 & 17 & 2.72 & 0.497 \\
\hline $\mathrm{T} 2$ & 29 & 45 & & & 28 & 35 & & \\
\hline $\mathrm{T} 3$ & 34 & 64 & & & 29 & 48 & & \\
\hline $\mathrm{T} 4$ & 20 & 46 & & & 13 & 32 & & \\
\hline \multicolumn{9}{|l|}{$\mathrm{N}$ stage } \\
\hline NO & 17 & 24 & 1.20 & 0.753 & 12 & 17 & 3.37 & 0.338 \\
\hline N1 & 29 & 42 & & & 24 & 28 & & \\
\hline $\mathrm{N} 2$ & 46 & 86 & & & 37 & 65 & & \\
\hline N3 & 14 & 27 & & & 8 & 22 & & \\
\hline \multicolumn{9}{|l|}{ AJCC stage } \\
\hline I & 5 & 6 & 0.71 & 0.870 & 5 & 4 & 4.38 & 0.224 \\
\hline II & 17 & 24 & & & 12 & 15 & & \\
\hline III & 52 & 84 & & & 44 & 64 & & \\
\hline IV & 32 & 65 & & & 20 & 49 & & \\
\hline \multicolumn{9}{|l|}{ Chemotherapy } \\
\hline No & 22 & 35 & 0.06 & 0.806 & 18 & 29 & 0.00 & 0.966 \\
\hline Yes & 84 & 144 & & & 63 & 103 & & \\
\hline \multicolumn{9}{|l|}{ IMRT } \\
\hline No & 61 & 100 & 0.08 & 0.782 & 31 & 57 & 0.50 & 0.480 \\
\hline Yes & 45 & 79 & & & 50 & 75 & & \\
\hline \multicolumn{9}{|l|}{ PLR } \\
\hline$\leq 112$ & 54 & 47 & 17.73 & $<0.001$ & 43 & 33 & 17.25 & $<0.001$ \\
\hline$>112$ & 52 & 132 & & & 38 & 99 & & \\
\hline \multicolumn{9}{|l|}{ NLR } \\
\hline$\leq 1.85$ & 72 & 20 & 72.31 & $<0.001$ & 56 & 10 & 88.96 & $<0.001$ \\
\hline$>1.85$ & 34 & 159 & & & 25 & 122 & & \\
\hline \multicolumn{9}{|l|}{ MLR } \\
\hline$\leq 0.29$ & 86 & 52 & 98.09 & $<0.001$ & 65 & 39 & 51.64 & $<0.001$ \\
\hline$>0.29$ & 20 & 127 & & & 16 & 93 & & \\
\hline
\end{tabular}

AJCC, American Joint Committee on Cancer; BMI, body mass index; IMRT, intensity-modulated radiotherapy; MLR, monocyte lymphocyte ratio.; NLR, neutrophil lymphocyte ratio; PLR, platelet lymphocyte ratio; SIRI, Systemic Inflammation Response Index. 
A

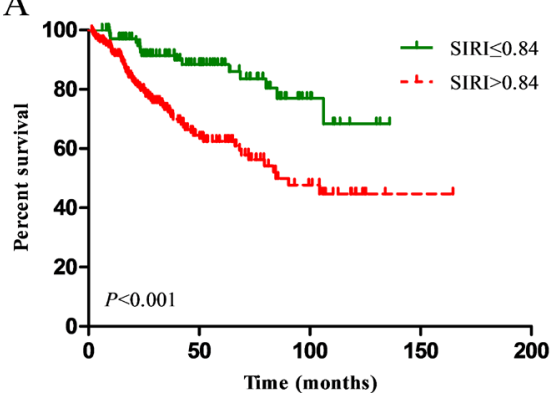

B

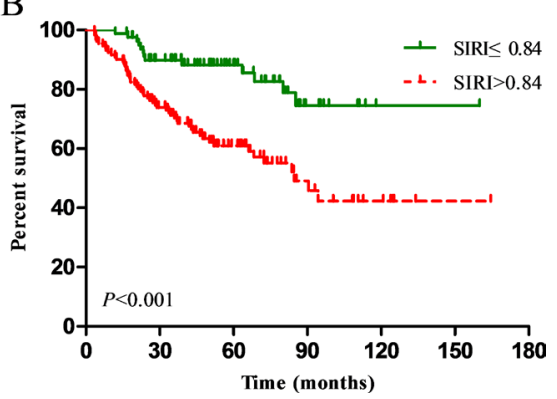

Figure 1 Kaplan-Meier survival curves for patients stratified based on Systemic Inflammation Response Index (SIRI) in the primary cohort (A) and validation cohort (B).

types of white blood cell indicators; the neutrophil lymphocyte ratio (NLR) and monocyte lymphocyte ratio (MLR) are considered to be independent prognostic factors for NPC, and their prognostic value is greater than that of only white blood cells. ${ }^{10-12}$ It has recently been reported that the SIRI, based on three types of white cells (peripheral neutrophils, monocytes and lymphocytes), is a new prognostic marker for pancreatic ${ }^{14}$ and liver cancer. ${ }^{15}$ However, the prognostic significance of the SIRI in NPC has not been reported. In this study, we first reported the prognostic value of the SIRI

Table 2 Univariate and multivariate cox regression analyses for overall survival in patients with nasopharyngeal carcinoma in the primary cohort

\begin{tabular}{|c|c|c|c|c|}
\hline \multirow[b]{2}{*}{ Variables } & \multicolumn{2}{|l|}{ Univariate analysis } & \multicolumn{2}{|c|}{ Multivariate analysis } \\
\hline & HR $(95 \% \mathrm{CI})$ & $P$ values & $\mathrm{HR}(95 \% \mathrm{Cl})$ & $P$ values \\
\hline \multicolumn{5}{|l|}{ Sex } \\
\hline Female versus male & 0.55 (0.31 to 0.97$)$ & $0.038^{*}$ & $0.68(0.38$ to 1.22$)$ & $0.194 \dagger$ \\
\hline \multicolumn{5}{|l|}{ Age } \\
\hline$\geq 50$ versus $<50$ & 1.69 (1.04 to 2.74$)$ & $0.035^{*}$ & 1.89 (0.98 to 3.78$)$ & $0.051 \dagger$ \\
\hline \multicolumn{5}{|l|}{ BMI } \\
\hline$>25$ versus $\leq 25$ & $0.78(0.45$ to 1.35$)$ & 0.376 & & \\
\hline T stage & & $0.003^{*}$ & & $0.002 * t$ \\
\hline $\mathrm{T} 1$ & Ref. & & Ref. & \\
\hline $\mathrm{T} 2$ & 2.56 (1.03 to 6.34$)$ & $0.043^{*}$ & 2.66 (1.06 to 6.70$)$ & $0.038^{*}$ \\
\hline T3 & 3.00 (1.23 to 7.29$)$ & $0.015^{*}$ & $3.52(1.43$ to 8.71$)$ & $0.006^{*}$ \\
\hline $\mathrm{T} 4$ & $5.02(2.05$ to 12.30$)$ & $<0.001^{*}$ & 5.56 (2.23 to 13.82$)$ & $<0.001^{*}$ \\
\hline $\mathrm{N}$ stage & & $0.005^{*}$ & & $0.003^{*} \dagger$ \\
\hline NO & Ref. & & Ref. & \\
\hline $\mathrm{N} 1$ & 3.97 (1.52 to 10.34$)$ & $0.005^{*}$ & 4.96 (1.88 to 13.14$)$ & $0.001^{*}$ \\
\hline N2 & 4.01 (1.56 to 10.34$)$ & $0.004^{*}$ & 4.90 (1.87 to 12.79$)$ & $0.001 *$ \\
\hline N3 & $6.93(2.42$ to 19.86$)$ & $<0.001^{*}$ & 7.59 (2.60 to 22.12$)$ & $<0.001^{*}$ \\
\hline \multicolumn{5}{|l|}{ Chemotherapy } \\
\hline Yes versus No & 1.05 (0.63 to 1.75$)$ & 0.846 & & \\
\hline \multicolumn{5}{|l|}{ IMRT } \\
\hline Yes versus No & 0.85 (0.52 to 1.37$)$ & 0.496 & & \\
\hline \multicolumn{5}{|l|}{ SIRI } \\
\hline$>0.84$ versus $\leq 0.84$ & 2.96 (1.69 to 5.20$)$ & $<0.001^{*}$ & 2.78 (1.57 to 4.92$)$ & $<0.001 * \dagger$ \\
\hline \multicolumn{5}{|l|}{ NLR } \\
\hline$>1.85$ versus $\leq 1.85$ & 2.08 (1.19 to 3.65$)$ & $0.011^{*}$ & $2.03(1.15$ to 3.60$)$ & $0.015^{*} \ddagger$ \\
\hline \multicolumn{5}{|l|}{ PLR } \\
\hline$>112$ versus $\leq 112$ & 1.82 (1.09 to 3.05$)$ & $0.023^{*}$ & $1.78(1.05$ to 3.00$)$ & $0.031 * \S$ \\
\hline \multicolumn{5}{|l|}{ MLR } \\
\hline$>0.29$ versus $\leq 0.29$ & 1.83 (1.16 to 2.89$)$ & $0.010^{*}$ & 1.61 (1.01 to 2.55$)$ & $0.046 * q$ \\
\hline
\end{tabular}

* represents a statistically significant difference.

†The variables (sex, age, T stage, $\mathrm{N}$ stage and SIRI) were tested in a multivariate analysis.

$¥$ The variables (sex, age, T stage, $N$ stage and NLR) were tested in a multivariate analysis.

$\S$ The variables (sex, age, T stage, N stag and PLR) were tested in a multivariate analysis.

ๆThe variables (sex, age, $T$ stage, $\mathrm{N}$ stage and MLR) were tested in a multivariate analysis.

BMI, body mass index; IMRT, intensity-modulated radiotherapy; MLR, monocyte lymphocyte ratio; NLR, neutrophil lymphocyte ratio; PLR, platelet lymphocyte ratio;

Ref, reference; SIRI, Systemic Inflammation Response Index. 
A

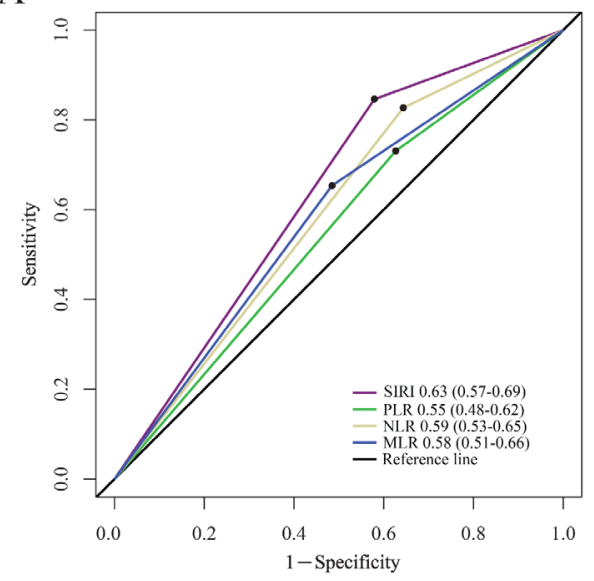

C

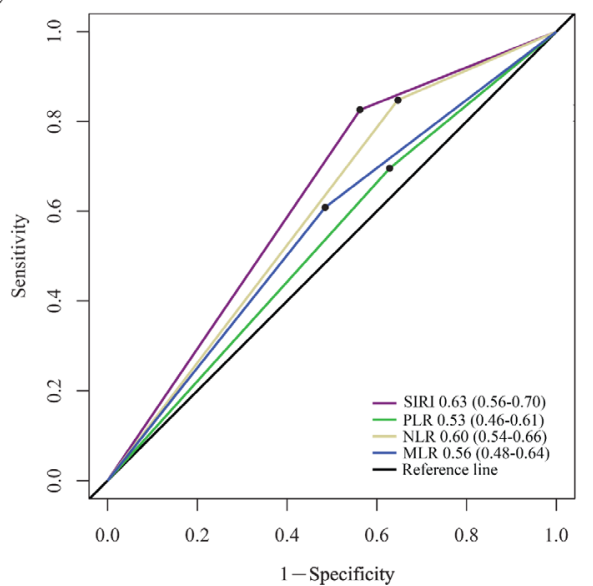

B

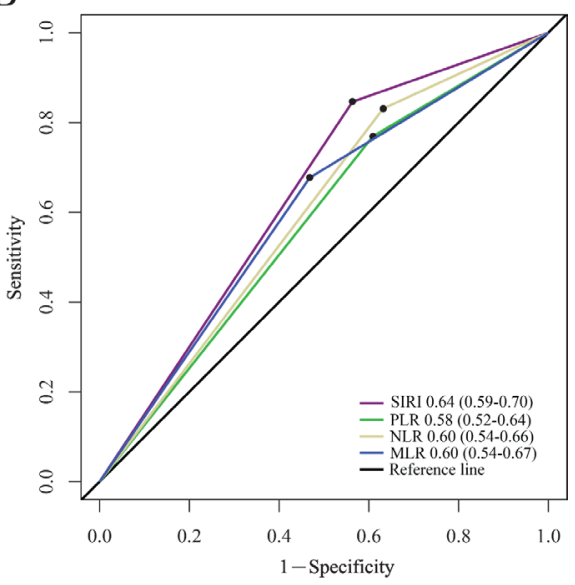

$\mathrm{D}$

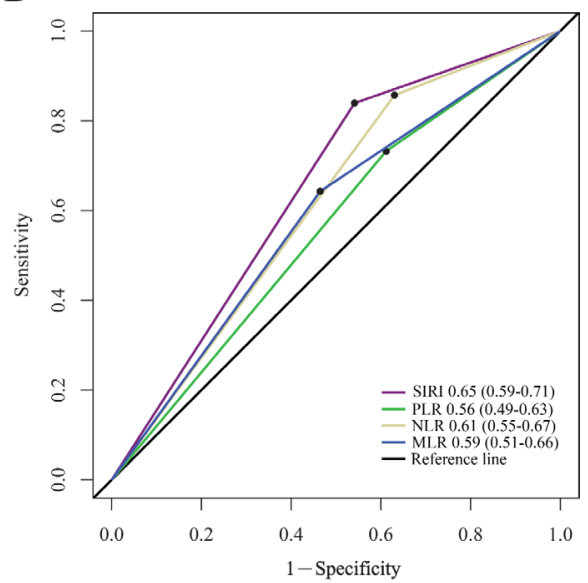

Figure 2 Predictive ability of the SIRI was compared with PLR, NLR and MLR by ROC curves at 3 years (A) and 5 years (B) in the primary cohort, and at 3 years (C) and 5 years (D) in the validation cohort. MLR, monocyte lymphocyte ratio; NLR, neutrophil lymphocyte ratio; PLR, platelet lymphocyte ratio; ROC, receiver operating characteristic; SIRI, Systemic Inflammation Response Index.

in NPC patients. In addition, whether the SIRI provides a better prognostic evaluation than conventional systemic inflammatory markers (NLR, platelet lymphocyte ratio (PLR) and MLR) was also assessed. Moreover, the prognostic effect of the SIRI in NPC was verified using an independent validation group. Finally, the nomogram based on clinicopathological features and the SIRI was established.

\section{MATERIALS AND METHODS}

\section{Patients}

We retrospectively recruited 285 patients who were newly identified as patients with NPC at the Third Affiliated Hospital of Soochow University from 2005 to 2012 as the primary cohort. To verify the prognostic value of the SIRI and nomogram, we used an independent data set of 213 patients with NPC who were diagnosed and treated at Nanjing General Hospital between 2005 and 2010. The patients were examined for neutrophil, platelet, lymphocyte and monocyte counts within 1 week before therapy. This study was conducted according to the Declaration of Helsinki. Written informed consent was obtained from all patients.

The peripheral blood of all patients was collected and tested for neutrophil, lymphocyte, platelet, and monocyte counts within 1 week before therapy. The SIRI, PLR,
NLR and MLR are defined as follows: SIRI=neutrophils*monocytes/lymphocytes; $\quad \mathrm{PLR}=$ platelets/lymphocytes; NLR=neutrophils/lymphocytes; MLR=monocytes/ lymphocytes. The optimal cut-off values for the SIRI $(\mathrm{SIRI} \leq 0.84, \mathrm{SIRI}>0.84), \mathrm{NLR}(\mathrm{NLR} \leq 1.85, \mathrm{NLR}>1.85)$, PLR (PLR $\leq 112, \quad$ PLR $>112)$ and MLR (MLR $\leq 0.29$, MLR>0.29) were determined by X-tile software (http:// www.tissuearray.org/rimmlab). ${ }^{16}$

\section{Statistical analysis}

The correlations between clinical factors and the systemic inflammatory indexes were analyzed by $\chi^{2}$ test. The KaplanMeier curves were plotted by the log-rank test. The nomogram was build based on the results of the multivariate analysis. We chose a backward stepwise selection process with the Akaike information criterion (AIC) for final model selection. ${ }^{17}$ Both the discrimination and calibration of these models were assessed to evaluate the nomogram performance. Analyses of time-dependent receiver operating characteristic (ROC) curves and the C-index were applied to compare the discrimination power between different models for OS. Statistical analyses were carried out by $\mathrm{R}$ software V.3.2.0 (http://www.r-project.org/) with Hmisc, rms, and survival ROC packages. 
Table 3 Univariate and multivariate cox regression analyses for overall survival in patients with nasopharyngeal carcinoma in the validation cohort

\begin{tabular}{|c|c|c|c|c|}
\hline \multirow[b]{2}{*}{ Variables } & \multicolumn{2}{|l|}{ Univariate analysis } & \multicolumn{2}{|l|}{ Multivariate analysis } \\
\hline & $\mathrm{HR}(95 \% \mathrm{Cl})$ & $P$ values & $\mathrm{HR}(95 \% \mathrm{Cl})$ & $P$ values \\
\hline \multicolumn{5}{|l|}{ Sex } \\
\hline Female versus male & 0.53 (0.29 to 1.00$)$ & $0.049^{*}$ & 0.76 (0.40 to 1.45$)$ & $0.408 \dagger$ \\
\hline \multicolumn{5}{|l|}{ Age } \\
\hline$\geq 50$ versus $<50$ & 1.77 (1.05 to 2.99$)$ & $0.034^{*}$ & 1.96 (0.95 to 4.52$)$ & $0.056 \dagger$ \\
\hline \multicolumn{5}{|l|}{ BMI } \\
\hline$>25$ versus $\leq 25$ & 0.72 (0.39 to 1.33$)$ & 0.294 & & \\
\hline T stage & & $0.004^{*}$ & & $0.002^{*} \dagger$ \\
\hline $\mathrm{T} 1$ & Ref. & & Ref. & \\
\hline $\mathrm{T} 2$ & 4.93 (1.15 to 21.08) & $0.032^{*}$ & 5.03 (1.17 to 21.76$)$ & $0.030^{*}$ \\
\hline T3 & $5.41(1.28$ to 22.91$)$ & $0.022^{*}$ & 5.37 (1.26 to 22.91$)$ & $0.023^{*}$ \\
\hline $\mathrm{T} 4$ & 10.24 (2.39 to 43.84$)$ & $<0.001^{*}$ & 11.48 (2.65 to 49.68$)$ & $0.001 *$ \\
\hline $\mathrm{N}$ stage & & $0.010^{*}$ & & $0.010^{*} \dagger$ \\
\hline No & Ref. & & Ref. & \\
\hline N1 & 2.90 (0.97 to 8.62$)$ & 0.056 & 3.08 (1.03 to 9.23$)$ & $0.044^{*}$ \\
\hline N2 & 3.28 (1.16 to 9.31$)$ & $0.026^{*}$ & 4.20 (1.46 to 12.07$)$ & $0.008^{*}$ \\
\hline N3 & 6.38 (2.06 to 19.71$)$ & $0.001^{*}$ & 6.64 (2.11 to 20.91$)$ & $0.001^{*}$ \\
\hline \multicolumn{5}{|l|}{ Chemotherapy } \\
\hline Yes versus no & 1.05 (0.60 to 1.82$)$ & 0.868 & & \\
\hline \multicolumn{5}{|l|}{ IMRT } \\
\hline Yes versus no & 0.92 (0.56 to 1.53$)$ & 0.756 & & \\
\hline \multicolumn{5}{|l|}{ SIRI } \\
\hline$>0.84$ versus $\leq 0.84$ & 3.10 (1.69 to 5.68$)$ & $<0.001^{*}$ & 2.91 (1.56 to 5.41$)$ & $0.001 * \dagger$ \\
\hline \multicolumn{5}{|l|}{ NLR } \\
\hline$>1.85$ versus $\leq 1.85$ & 2.82 (1.44 to 5.52$)$ & $0.003^{*}$ & 2.99 (1.51 to 5.95$)$ & $0.002 * \ddagger$ \\
\hline \multicolumn{5}{|l|}{ PLR } \\
\hline$>112$ versus $\leq 112$ & 1.57 (1.03 to 2.70$)$ & $0.042^{*}$ & 1.74 (1.01 to 3.03$)$ & $0.048 * \S$ \\
\hline \multicolumn{5}{|l|}{ MLR } \\
\hline$>0.29$ versus $\leq 0.29$ & 1.77 (1.08 to 2.90$)$ & $0.024^{*}$ & $1.54(0.93$ to 2.54$)$ & 0.0939 \\
\hline
\end{tabular}

* represents a statistically significant difference.

tThe variables (sex, age, T stage, $\mathrm{N}$ stage and SIRI) were tested in a multivariate analysis.

$¥$ The variables (sex, age, T stage, N stage and NLR) were tested in a multivariate analysis.

$\S$ The variables (sex, age, T stage, $\mathrm{N}$ stage and PLR) were tested in a multivariate analysis.

१The variables (sex, age, T stage, $\mathrm{N}$ stage and MLR) were tested in a multivariate analysis.

BMI, body mass index; IMRT, intensity-modulated radiotherapy; MLR, monocyte lymphocyte ratio; NLR, neutrophil lymphocyte ratio; PLR, platelet lymphocyte ratio; Ref, reference;

SIRI, Systemic Inflammation Response Index.

\section{RESULTS}

\section{Patients' characteristics}

Two hundred and eighty-five patients with NPC initially treated at the Third Affiliated Hospital of Soochow University were included in the primary cohort, including 210 male and 75 female patients in the $22-80$ age range. The 3-year and 5-year OS rate were $79.4 \%$ and $71.0 \%$, respectively. There were 213 patients in the validation cohort. The 3-year and 5 -year OS rate were $77.7 \%$ and $70.1 \%$, respectively.

Correlations between the SIRI and clinical patient parameters are shown in table 1. Patients with a SIRI value $>0.84$ were more likely to be male in the primary cohort $(\mathrm{p}=0.011$, table 1$)$. We also examined the association between the SIRI and other indexes: the PLR, NLR and MLR (table 1). The results showed that there was a correlation between SIRI and these inflammation prognostic indexes (all $\mathrm{p}<0.001$ ). The validation cohort showed the same results (table 1).
Points

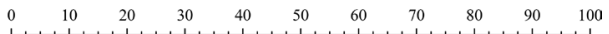

SIRI

T stage

$\mathrm{N}$ stage

Total Points

3-year survival

5-year survival
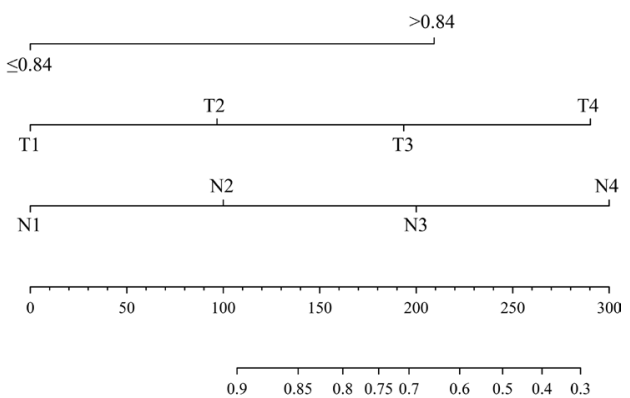

Figure 3 Evaluation of nomogram integrated systemic inflammation response index (SIRI) and clinicopathological factors in patients with nasopharyngeal carcinomas. 


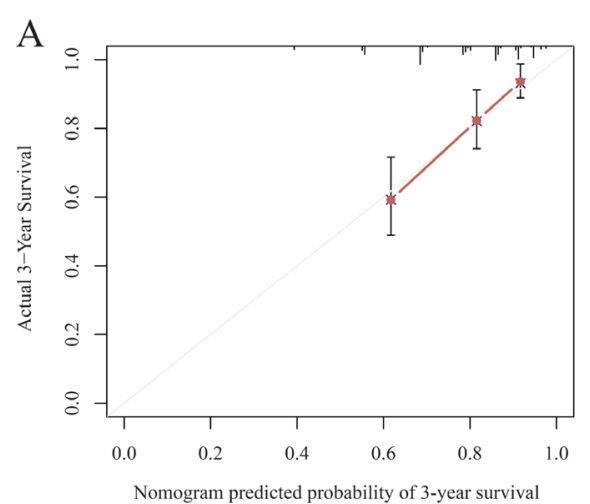

$\mathrm{C}$

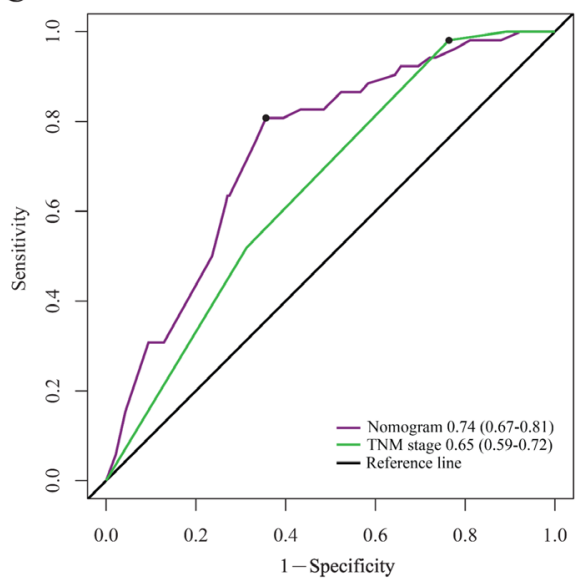

B

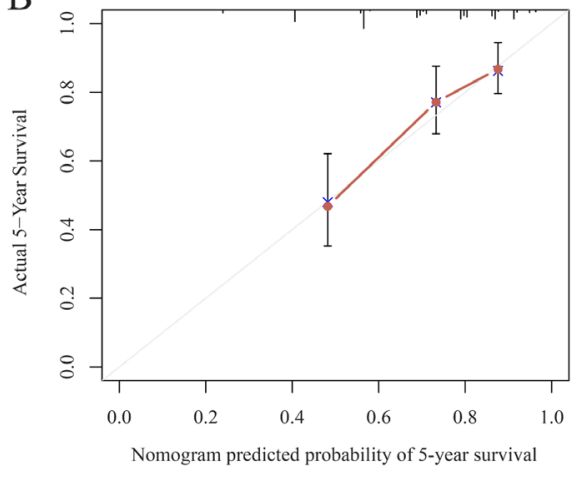

$\mathrm{D}$

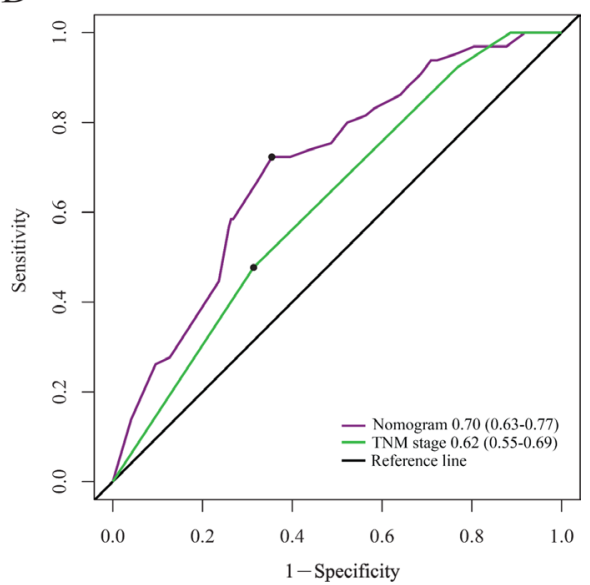

Figure 4 The calibration curve for predicting patient survival at 3 year (A) and 5 year (B) in the primary cohort. Time-dependent receiver operating characteristic curves by nomogram and tumor-node-metastasis (TNM) staging system for 3-year (C) and 5-year (D) overall survival in the primary cohort.

\section{Prognostic significance of the SIRI}

The OS of patients with higher SIRI values $(>0.84)$ was significantly inferior to that of patients with lower SIRI values $(\leq 0.84)$ in the primary cohort $(\mathrm{p}<0.001$, figure $1 \mathrm{~A})$. Age, sex, T stage, N stage, SIRI, PLR, NLR and MLR significantly affected the OS in patients with NPC in univariate analysis, while body mass index (BMI), chemotherapy and intensity-modulated radiotherapy (IMRT) were not associated with prognosis (table 2). Since the SIRI, NLR, PLR and MLR variables were highly correlated, four separate multivariate models were established to avoid problems of multicollinearity. Multivariate analyses showed that T stage, $\mathrm{N}$ stage, SIRI, NLR, PLR and MLR were independent risk factors for OS of patients with NPC in the primary cohort (table 2). In the subsequent analyses, the area under the ROC curve for the SIRI was significantly larger than that for the NLR, PLR and MLR at 3 and 5 years, indicating that the prognostic value of the SIRI for NPC was significantly superior to that of the NLR, PLR and MLR (figure 2A,B). These results were confirmed in the validation cohort (table 3, figures 1B, 2C,D).

\section{Nomogram development and validation}

In order to find a best-fit model, AIC in Cox proportional hazards regression modeling was used. Finally, the nomogram that integrated the $\mathrm{T}$ stage, $\mathrm{N}$ stage and SIRI was used to predict 3-year and 5-year OS rates in the primary cohort (figure 3). In the intragroup validation, the calibration plot for the 3-year and 5-year survival rates were very satisfied (figure 4A,B). The C-index for OS prediction was 0.76 , which was significantly higher than that of TNM staging 0.70. In addition, the time-dependent ROC curve showed the same results (figure 4C,D). So, our nomogram showed better accuracy for predicting survival in the primary cohort. In the inter-group validation, the nomogram was still good. The calibration curve revealed that the 3-year and 5-year survival rates predicted by the nomogram matched well with the actual observations (figure 5A,B). In the validation cohort, the $\mathrm{C}$-index of the nomogram for survival prediction was 0.78 (95\% CI 0.70 to 0.86$)$, which was significantly higher than that of TNM staging $(0.69)(p<0.001)$. ROC curve analyses also suggested similar results (figure 5C,D). These results confirmed that the nomogram is a more accurate and effective tool for predicting the survival of patients with NPC.

\section{DISCUSSION}

In this study, we conducted a retrospective analysis from two centers; the data confirm that a high SIRI value is a poor prognostic factor for NPC. The SIRI has been recently reported in pancreatic cancer and liver cancer. The SIRI in NPC has not been investigated. This study confirmed the prognostic effect of the SIRI for NPC for the first time, and its prognostic value is significantly greater than 
that of traditional systemic inflammatory markers. The results of this study are consistent with those reported in pancreatic cancer and liver cancer; consequently, the SIRI may be another reliable and convenient biomarker that represents the systemic inflammatory response. In addition, we created and validated a new prognostic nomogram that integrated clinicopathological factors and the SIRI. This nomogram can sufficiently predict the prognosis of patients with NPC. In our study, the accuracy of the nomogram for survival prediction was as high as 0.76 , which was significantly superior to that of the TNM staging system. Compared with the TNM staging system, this ROC curve can predict the 3-year and 5-year OS more sensitively and specifically. These results were validated using a group of independent external data. Therefore, we can see that the nomogram can reliably predict the survival of patients with NPC and could be used to develop personalized treatment programmes.

A large amount of evidence proves that the systemic immune response in patients play an important prognostic biomarker. The inflammatory response in the microenvironment can induce tumor occurrence, development, and metastasis. ${ }^{718}$ Virchow et al described the interaction between inflammation and tumors as early as a century ago. ${ }^{6}$ NPC is typically characterized by the infiltration of leucocyte subtypes, among which monocytes, neutrophils and lymphocytes play important roles in inflammation. Monocytes, neutrophils and lymphocytes, or

A

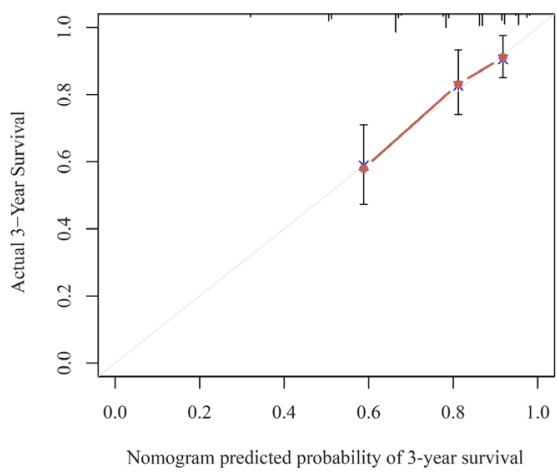

$\mathrm{C}$

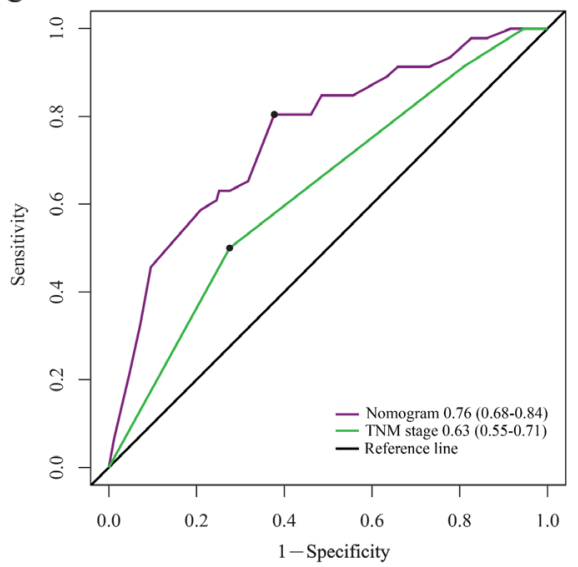

indicators using their combinations (the NLR and MLR), are associated with the prognosis of NPC. ${ }^{10-12}$ The antitumor mechanisms of the three cell types are as follows: (1) tumor-associated macrophages (TAMs) derived from mononuclear cell precursors in circulating blood are recruited to tumor tissues through chemotaxis, ${ }^{19}$ which is an important part of the inflammatory infiltration of malignant tumors. Tumor-promoting TAMs regulate the tumor microenvironment through a variety of mechanisms, including the regulation of senescence, promotion of extracellular matrix remodeling, promotion of cell proliferation and promotion of vascular and lymphatic vessel production. ${ }^{2021}$ The number of mononuclear cells in the cycle may reflect the formation and level of TAMs, so monocytes are considered to be negative markers for tumors. (2) Large amounts of arginase, nitric oxide and ROS secreted by circulating neutrophils can lead to $\mathrm{T}$ cell activation disorders. ${ }^{22}$ At the same time, it has been reported that the induced circulating neutrophils can also produce vascular endothelial growth factor, which can cause tumor angiogenesis. ${ }^{23}$ In addition, neutrophils can promote the transfer of tumor cells. (3) Compared with monocytes and neutrophils, which can promote tumor activity, lymphocytes play an important antitumor role in immunoregulation; lymphocytes destroy tumor cells and related metastases. ${ }^{24} 25$ Although the monocyte, neutrophil and lymphocyte counts in the cycle can be used as prognostic factors, this study confirms that the SIRI,

B

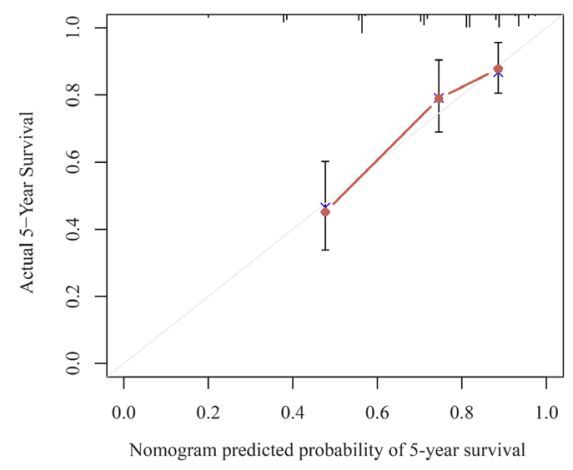

$\mathrm{D}$

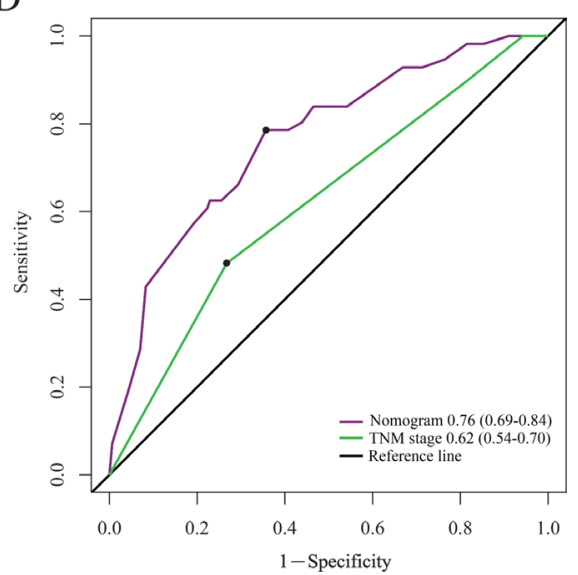

Figure 5 The calibration curve for predicting patient survival at 3 year (A) and 5 year (B) in the validation cohort. Time-dependent receiver operating characteristic curves by nomogram and tumor-node-metastasis (TNM) staging system for 3-year (C) and 5-year (D) overall survival in the validation cohort. 
which combines the three cell types, has a stronger prognostic value. Although the lymphocyte count is a good prognostic factor, the monocyte and neutrophil counts are poor prognostic factors; the SIRI combines the above variables to expand their prognostic value. At the same time, the interactions among the three leucocyte subtypes may also enhance the prognostic value of the indicator.

Recently, several studies have reported that a nomogram combining the systemic inflammatory response biomarkers can better predict the prognosis of multiple tumors than traditional staging systems. ${ }^{10}{ }^{26-28}$ Regarding NPC, Li et al established a nomogram based on systemic inflammation; the nomogram included the MLR, NLR and clinicopathological parameters. ${ }^{10}$ The SIRI was selected for our nomogram, which is more convenient, has fewer parameters and is easier to use than Li's nomogram. In addition, Li et al.'s nomogram was not subjected to external validation, whereas our nomogram was externally validated using other patients. So, our nomogram is more reliable. However, there are still some shortcomings regarding this research. First, this analysis did not include EBV-DNA data, mainly because the EBV-DNA test was not routinely performed in our hospital until 2006. Second, this is a retrospective study, there may be selective bias in data collection. Finally, at that time, not all the patients in this analysis received IMRT, which is a routine radiotherapy technology used for NPC nowadays.

In conclusion, the pretreatment SIRI value is a novel systemic inflammatory marker for patients with NPC, and its value is significantly more than that of conventional systemic inflammatory markers. In addition, our nomogram combining clinicopathologic factors and the SIRI can accurately predict the survival of patients with NPC. The SIRI has important value for assessing the prognosis of patients with NPC and developing individualized treatment strategies.

Contributors YC, WJ and WG conceived and designed the study and helped to draft the manuscript. DX, JC and GX performed the data collection. WY and $J J C$ performed the statistical analysis. All authors read and critically revised the manuscript for intellectual content and approved the final manuscript.

Funding The authors have not declared a specific grant for this research from any funding agency in the public, commercial or not-for-profit sectors.

Competing interests None declared.

Patient consent Not required.

Ethics approval The Third Affiliated Hospital of Soochow University.

Provenance and peer review Not commissioned; externally peer reviewed.

Open access This is an open access article distributed in accordance with the Creative Commons Attribution Non Commercial (CC BY-NC 4.0) license, which permits others to distribute, remix, adapt, build upon this work noncommercially, and license their derivative works on different terms, provided the original work is properly cited, an indication of whether changes were made, and the use is non-commercial. See: http://creativecommons.org/ licenses/by-nc/4.01.

\section{REFERENCES}

1 Chua MLK, Wee JTS, Hui EP, et al. Nasopharyngeal carcinoma. Lancet 2016;387:1012-24.
2 Jia WH, Qin HD. Non-viral environmental risk factors for nasopharyngeal carcinoma: a systematic review. Semin Cancer Biol 2012;22:117-26.

3 Chan AT. Nasopharyngeal carcinoma. Ann Oncol 2010;21 Suppl 7:vii308-vii312.

4 Ludwig JA, Weinstein JN. Biomarkers in cancer staging, prognosis and treatment selection. Nat Rev Cancer 2005;5:845-56.

5 Lu H, Ouyang W, Inflammation HC. a key event in cancer development. Mol Cancer Res 2006:4:221-33.

6 Balkwill F, Mantovani A. Inflammation and cancer: back to Virchow? Lancet 2001;357:539-45.

7 Hanahan D, Weinberg RA. Hallmarks of cancer: the next generation. Cell 2011;144:646-74.

8 Gourzones C, Barjon C, Busson P. Host-tumor interactions in nasopharyngeal carcinomas. Semin Cancer Biol 2012;22:127-36.

9 Lin JC, Wang WY, Chen KY, et al. Quantification of plasma Epstein-Barr virus DNA in patients with advanced nasopharyngeal carcinoma. N Eng/ J Med 2004;350:2461-70.

10 Li XH, Chang H, Xu BQ, Xh L, Bq X, et al. An inflammatory biomarker-based nomogram to predict prognosis of patients with nasopharyngeal carcinoma: an analysis of a prospective study. Cancer Med 2017;6:310-9.

11 Chua ML, Tan SH, Kusumawidjaja G, et al. Neutrophil-to-lymphocyte ratio as a prognostic marker in locally advanced nasopharyngeal carcinoma: a pooled analysis of two randomised controlled trials. Eur J Cancer 2016;67:119-29.

12 Sun W, Zhang L, Luo M, et al. Pretreatment hematologic markers as prognostic factors in patients with nasopharyngeal carcinoma: Neutrophil-lymphocyte ratio and platelet-lymphocyte ratio. Head Neck 2016;38 Suppl 1(Suppl 1):E1332-E1340.

13 Su L, Zhang M, Zhang W, et al. Pretreatment hematologic markers as prognostic factors in patients with nasopharyngeal carcinoma: A systematic review and meta-analysis. Medicine 2017;96:e6364.

14 Qi Q, Zhuang L, Shen Y, et al. A novel systemic inflammation response index (SIRI) for predicting the survival of patients with pancreatic cancer after chemotherapy. Cancer 2016;122:2158-67.

15 Xu L, Yu S, Zhuang L, et al. Systemic inflammation response index (SIRI) predicts prognosis in hepatocellular carcinoma patients. Oncotarget 2017:8:34954-60.

16 Camp RL, Dolled-Filhart M, Rimm DL. X-tile: a new bio-informatics tool for biomarker assessment and outcome-based cut-point optimization. Clin Cancer Res 2004;10:7252-9.

17 Harrell FE, Lee KL, Mark DB. Multivariable prognostic models: issues in developing models, evaluating assumptions and adequacy, and measuring and reducing errors. Stat Med 1996;15:361-87.

18 Landskron G, De la Fuente M, Thuwajit P, et al. Chronic inflammation and cytokines in the tumor microenvironment. J Immunol Res 2014;2014:1-19.

19 Galdiero MR, Garlanda C, Jaillon S, et al. Tumor associated macrophages and neutrophils in tumor progression. J Cell Physiol 2013;228:1404-12.

20 Lin EY, Li JF, Gnatovskiy L, et al. Macrophages regulate the angiogenic switch in a mouse model of breast cancer. Cancer Res 2006;66:11238-46.

21 Clear AJ, Lee AM, Calaminici M, et al. Increased angiogenic sprouting in poor prognosis $\mathrm{FL}$ is associated with elevated numbers of CD163+ macrophages within the immediate sprouting microenvironment. Blood 2010;115:5053-6.

22 Müller I, Munder M, Kropf P, et al. Polymorphonuclear neutrophils and T lymphocytes: strange bedfellows or brothers in arms? Trends Immunol 2009:30:522-30.

23 Kusumanto YH, Dam WA, Hospers GA, et al. Platelets and granulocytes, in particular the neutrophils, form important compartments for circulating vascular endothelial growth factor. Angiogenesis 2003;6:283-7.

24 Mantovani A, Allavena P, Sica A, et al. Cancer-related inflammation. Nature 2008:454:436-44

25 Ferrone C, Dranoff G. Dual roles for immunity in gastrointestinal cancers. J Clin Oncol 2010;28:4045-51.

26 Shao Y, Ning Z, Chen J, et al. Prognostic nomogram integrated systemic inflammation score for patients with esophageal squamous cell carcinoma undergoing radical esophagectomy. Sci Rep 2015;5:18811.

27 Deng Q, He B, Liu X, et al. Prognostic value of pre-operative inflammatory response biomarkers in gastric cancer patients and the construction of a predictive model. J Trans/ Med 2015;13:66.

28 Chen Z, Shao Y, Yao H, et al. Preoperative albumin to globulin ratio predicts survival in clear cell renal cell carcinoma patients. Oncotarget 2017;8:48291-302. 